\title{
Non-Hermitian impurities in Dirac systems
}

\author{
P. O. Sukhachov $\oplus^{1, *}$ and A. V. Balatsky $\oplus^{1,2, \dagger}$ \\ ${ }^{1}$ Nordita, KTH Royal Institute of Technology and Stockholm University, Roslagstullsbacken 23, SE-106 91 Stockholm, Sweden \\ ${ }^{2}$ Department of Physics, University of Connecticut, Storrs, Connecticut 06269, USA
}

(Received 31 December 2019; accepted 21 February 2020; published 17 March 2020)

\begin{abstract}
Quasiparticle states in Dirac systems with complex impurity potentials are investigated. It is shown that an impurity site with loss leads to a nontrivial distribution of the local density of states (LDOS). While the real part of defect potential induces a well-pronounced peak in the density of states (DOS), the DOS is either weakly enhanced at small frequencies or even forms a peak at the zero frequency for a lattice in the case of non-Hermitian impurity. As for the spatial distribution of the LDOS, it is enhanced in the vicinity of impurity but shows a dip at a defect itself when the potential is sufficiently strong. The results for a two-dimensional hexagonal lattice demonstrate the characteristic trigonal-shaped profile for the LDOS. The latter acquires a double-trigonal pattern in the case of two defects placed at neighboring sites. The effects of non-Hermitian impurities could be tested both in photonic lattices and certain condensed matter setups.
\end{abstract}

DOI: 10.1103/PhysRevResearch.2.013325

\section{INTRODUCTION}

Non-Hermitian systems that are governed by nonHermitian operators [1,2] are the subject of a significant interest. One of the most studied cases are the $\mathcal{P} \mathcal{T}$-symmetric Hamiltonians (see Refs. [3-7] for reviews). As was first suggested in Refs. [8,9], the combined action of the parityinversion $\mathcal{P}$ and time-reversal $\mathcal{T}$ symmetries allows for purely real spectra of non-Hermitian operators. Gain and loss for such $\mathcal{P} \mathcal{T}$-symmetric systems are compensated.

Recently, the concept of non-Hermitian and, in particular, $\mathcal{P} \mathcal{T}$-symmetric systems, spread beyond quantum mechanics to metamaterials and artificial structures. Such a surge of interest is explained by experimental realizations of various non-Hermitian phases in optical waveguides and lattices (for a recent brief comment on the prospects of the non-Hermitian optics, see Ref. [10]). Non-Hermitian Hamiltonians are able to capture certain aspects of open systems with gain and loss. Moreover, the concept of topology is also applied to the non-Hermitian domain [11-17]. For example, the nonHermitian boundary modes and the bulk-boundary correspondence [17-25] are actively investigated.

Photonic lattices proved to be a particularly fertile ground to investigate topologically nontrivial phases in nonHermitian systems [26-30]. These lattices can be effectively used also to probe various disorder and transport effects. For example, the Anderson localization was experimentally observed in a two-dimensional (2D) photonic lattice in Ref. [31].

\footnotetext{
*pavlo.sukhachov@su.se

†avb@nordita.org
}

Published by the American Physical Society under the terms of the Creative Commons Attribution 4.0 International license. Further distribution of this work must maintain attribution to the author(s) and the published article's title, journal citation, and DOI. Funded by Bibsam.
Furthermore, optical waveguides and photonic lattices allow for the realization of the so-called photonic graphene, which is discussed, for instance, in Refs. [32-38]. These examples point to the growing focus on artificial topological and Dirac materials.

The notion of Dirac materials became widely spread in modern condensed matter. These materials represent a special class of two- and three-dimensional (3D) systems whose quasiparticles demonstrate relativistic-like behavior. Among them are graphene [39-44], topological insulators [44-48], 3D Dirac and Weyl semimetals [44,49-51], etc. The valence and conduction bands of Dirac materials touch in isolated Dirac points of the Brillouin zone allowing one to apply the Dirac equation for the description of quasiparticle properties. This leads to many interesting effects including, in particular, nontrivial manifestations of disorder.

It is well known that impurities and defects significantly affect the properties of various materials. In particular, they are crucial for most of transport properties and play an important role in semiconductor physics. The manifestations of disorder are not limited to hindering quasiparticle propagation and broadening of spectral lines only. Defects enable new phenomena and effects as well. The latter include the appearance of additional electronic states known as impurity resonances (for a review, see Refs. [52-54]). As was noted by I. M. Lifshitz [55,56], the presence of even a small number of defects can lead to a noticeable spectrum rearrangement near van Hove singularities. In the case of relativistic-like spectrum, the latter correspond to Dirac points. Due to a large density of states (DOS) in conventional metals, the modification caused by impurity resonances is usually negligible. On the other hand, the DOS in Dirac systems has the characteristic linear (2D) or quadratic (3D) dependence on energy and vanishes in a Dirac point. This results in the resonance peak near a minimum in the DOS [57-62]. Note that the so-called unitary limit, where the impurity potential is infinitely large, was considered in Refs. [57,58]. In this case, the presence of the 
resonance state at zero frequency is allowed by a nonzero DOS due to finite size effects. The other hallmark feature of impurity resonances is the specific pattern of the local DOS (LDOS), whose trigonal form was observed via the scanning tunneling microscopy (STM) in graphene [63-66].

An impurity potential in condensed matter systems can be either attractive or repulsive and is usually taken to be real. For example, vacancies and ad-atoms like hydrogen are typical impurities in graphene [57-62,67,68]. Recent interest in non-Hermitian models opens up a new set of questions on the role of non-Hermitian defects in condensed matter setting including photonic and cold atom platforms. In particular, non-Hermitian random disorder in 2D and 3D systems was recently investigated in Refs. [69,70].

The focus of this study is the non-Hermitian effects in Dirac matter, e.g., in photonic graphene. Motivated by experimental [31,71] and recent theoretical $[38,69,70,72,73]$ studies in optical non-Hermitian systems, we investigate the nonHermitian defects in 2D and 3D Dirac systems. In particular, we show how the presence of the imaginary part of defect potential affects the spatial distribution of the LDOS and changes the frequency dependence of the DOS. Our analytical results are supported by tight-binding calculations for a $2 \mathrm{D}$ hexagonal lattice. Among the key findings is the redistribution of the LDOS for a dissipative potential. Like in the case of usual impurities, the LDOS demonstrates a peak near the defect (continuum model) or a trigonal profile (lattice). This is particularly interesting since no well-pronounced peaks appear in the frequency dependence of the DOS in this case. In that regard, we point the related but different proposal in Ref. [72], where the formation of bound states inside the band gap due to a $\mathcal{P} \mathcal{T}$-symmetric imaginary potential in strongly coupled bilayer lattices was considered. In addition, unlike Refs. $[69,70]$ where a randomly distributed non-Hermitian disorder was studied, we concentrate on the case of a few impurities with a strong potential. We also discuss experimental feasibility of non-Hermitian impurity in electronic and photonic systems.

The paper is organized as follows. In Sec. II A, we introduce a continuum model and the key details of the formalism. The results for the frequency and spatial dependence of the LDOS are presented in Sec. IIB. Section III is devoted to the $2 \mathrm{D}$ hexagonal lattice model. Finally, the conclusions are presented in Sec. IV. Additional results are presented in a few appendices. In particular, the case of 3D continuum Dirac Hamiltonian is discussed in Appendix A. The spatial dependence of the LDOS in the lattice model for an imaginary potential is analyzed in Appendix B. The evolution of the LDOS with the lattice size is discussed in Appendix C. Through this study, we set $\hbar=k_{B}=1$.

\section{CONTINUUM DIRAC MODEL}

\section{A. General definitions}

In this section, we formulate the continuum model of gapless Dirac systems and discuss the effects of a single impurity. The results for a simple tight-binding Hamiltonian of a 2D hexagonal lattice are presented in Sec. III.
The low-energy Dirac Hamiltonian reads as

$$
H_{0}=\int d^{n} r \bar{\Psi}(\mathbf{r})\left[-i v_{F}(\boldsymbol{\gamma} \cdot \nabla)\right] \Psi(\mathbf{r}),
$$

where $\bar{\Psi}=\Psi^{\dagger} \gamma^{0}$ is the conventional Dirac-conjugate bispinor, $\gamma$ is the vector of the spatial Dirac $\gamma$ matrices, $v_{F}$ is the Fermi velocity (or its analog in optical lattice setups), and $n=2,3$ is the spatial dimension of the system.

The impurity is described via the following interaction term:

$$
H_{\mathrm{int}}=\int d^{n} r \bar{\Psi}(\mathbf{r}) U(\mathbf{r}) \Psi(\mathbf{r}),
$$

where the short-range impurity potential is $U(\mathbf{r})=$ $U \delta(\mathbf{r}) \gamma^{0}+\Delta \delta(\mathbf{r})$. Here the first term corresponds to a spinless potential that does not intermix valleys and the term $\Delta$ plays the role of the Dirac mass that mixes spinors from different valleys. It is important to emphasize that the impurity potential $U$ in the case under consideration is complex. While its negative imaginary part corresponds to a dissipative or a lossy defect, the positive one allows for an amplification or a gain. In what follows, we will primarily focus on the case of lossy defects, which can be routinely realized in optical lattices.

To investigate effects of impurity and the formation of impurity resonances, we employ the standard $T$-matrix formalism [74]. Since the only non-Hermitian part of the Hamiltonian is related to the impurity potential, the eigenvalues of the noninteracting Hamiltonian $H_{0}$ are real. Therefore, one can define the following bare retarded Green's function in the frequency-momentum space:

$$
G_{0}^{\mathrm{R}}(\omega, \mathbf{k})=\frac{\omega \gamma^{0}-v_{F}(\boldsymbol{\gamma} \cdot \mathbf{k})}{\omega^{2}-v_{F}^{2} k^{2}+i 0 \operatorname{sgn}(\omega)} .
$$

The full Green's function in the $T$-matrix approach is built upon the retarded Green's functions of noninteracting quasiparticles and reads as

$$
G\left(\omega, \mathbf{k}, \mathbf{k}^{\prime}\right)=\delta_{\mathbf{k}, \mathbf{k}^{\prime}} G_{0}^{\mathrm{R}}(\omega, \mathbf{k})+G_{0}^{\mathrm{R}}(\omega, \mathbf{k}) T_{\mathbf{k}, \mathbf{k}^{\prime}} G_{0}^{\mathrm{R}}\left(\omega, \mathbf{k}^{\prime}\right) .
$$

The $T$ matrix for a local disorder itself is defined as $T_{\mathbf{k}, \mathbf{k}^{\prime}}=$ $\delta_{\mathbf{k}, \mathbf{k}^{\prime}} T$, where

$$
T=\left(U \gamma^{0}+\Delta\right)\left[1+\sum_{\mathbf{k}} G_{0}^{\mathrm{R}}(\omega, \mathbf{k}) T\right] .
$$

From the latter equation, it is straightforward to obtain the following $T$ matrix:

$$
T=\left[1-\left(U \gamma^{0}+\Delta\right) \sum_{\mathbf{k}} G_{0}^{\mathrm{R}}(\omega, \mathbf{k})\right]^{-1}\left(U \gamma^{0}+\Delta\right),
$$

where $\sum_{\mathbf{k}}=\int d^{n} k /(2 \pi)^{n}$. According to Eq. (4), the poles of the $T$ matrix are also the poles of the full Green's function $G\left(\omega, \mathbf{k}, \mathbf{k}^{\prime}\right)$, which give bound states and resonances. As long as we consider lossy impurities, the analytical properties of the $T$ matrix remain unchanged. As to the case of defects with gain, it requires a special treatment and will be addressed elsewhere. 

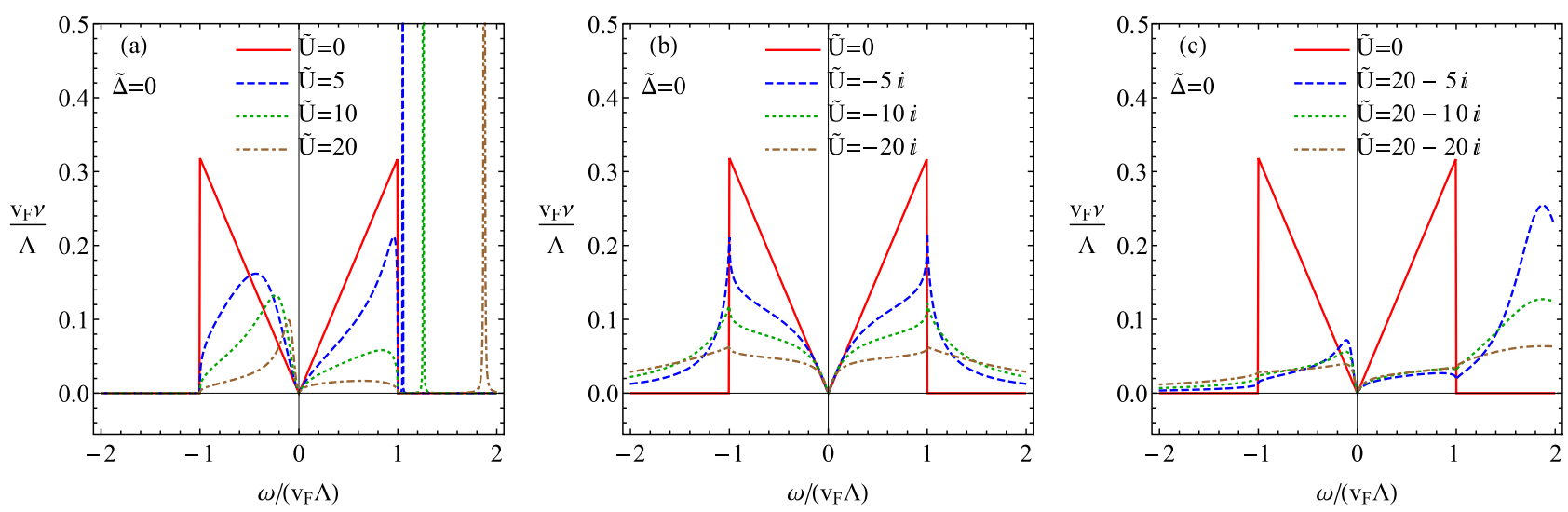

FIG. 1. The LDOS at the impurity site $v=v(\omega, \mathbf{0})$ for real (a), imaginary (b), and complex (c) impurity potentials as a function of the frequency $\omega$. Red solid, blue dashed, green dotted, and brown dot-dashed lines correspond to different disorder strengths shown in the corresponding legends. In addition, $\tilde{U}=U \Lambda / v_{F}$ and $\Delta=0$.

Bound states and resonances correspond to sharp features in the frequency and position dependencies of the LDOS [52-54]. The LDOS is defined as

$$
v(\omega, \mathbf{r})=-\frac{1}{\pi} \operatorname{Im} \operatorname{tr}\left[\gamma^{0} G^{\mathrm{R}}(\omega, \mathbf{r}, \mathbf{r})\right],
$$

where the full Green's function in the frequency-coordinate representation is

$$
G^{\mathrm{R}}\left(\omega, \mathbf{r}, \mathbf{r}^{\prime}\right)=G_{0}^{\mathrm{R}}\left(\omega, \mathbf{r}-\mathbf{r}^{\prime}\right)+G_{0}^{\mathrm{R}}(\omega, \mathbf{r}) T G_{0}^{\mathrm{R}}\left(\omega,-\mathbf{r}^{\prime}\right) .
$$

It is clear that the translation invariance is lost due to the presence of defect. Without the loss of generality, the position of the latter was set to $\mathbf{0}$. The first term in the above equation corresponds to the background LDOS, which is independent on $\mathbf{r}$ and reads as

$$
v_{0}=\frac{|\omega|}{\pi v_{F}^{2}} \theta\left(v_{F} \Lambda-|\omega|\right),
$$

for a 2D Dirac Hamiltonian.

\section{B. Impurity resonances and density of states}

Let us apply the formalism described in Sec. II A and consider both frequency and spatial dependence of the LDOS. Further, we demonstrate the effects of impurities with complex potential for the 2D Dirac phase. The results for the 3D case are presented in Appendix A.

The denominator of the $T$ matrix defined in Eq. (6) reads as

$$
\begin{aligned}
1- & \left(U \gamma^{0}+\Delta\right) \int \frac{d^{2} k}{(2 \pi)^{2}} G_{0}^{\mathrm{R}}(\omega, \mathbf{k}) \\
= & 1+\left(U+\Delta \gamma^{0}\right) \frac{1}{4 \pi v_{F}^{2}}\left[\omega \ln \left|1-\frac{v_{F}^{2} \Lambda^{2}}{\omega^{2}}\right|\right. \\
& \left.+i \pi|\omega| \theta\left(v_{F} \Lambda-|\omega|\right)\right] .
\end{aligned}
$$

Zeroes of the corresponding denominator determine the resonance and bound states. [It is worth noting that the real part of this equation is often referred to as the Lifshitz equation.] By using Eqs. (7) and (8), it is straightforward to derive the $\operatorname{LDOS} v(\omega, \mathbf{r})$. We present the latter as a function of the frequency $\omega$ at $\mathbf{r}=\mathbf{0}$ in Fig. 1 for a few values of real, imaginary (lossy), and complex potentials. Note that in order to show the presence of the bound states at $|\omega|>\Lambda$, we added a small imaginary part to the denominator of the $T$ matrix, i.e., $\theta\left(v_{F} \Lambda-|\omega|\right) \rightarrow \theta\left(v_{F} \Lambda-|\omega|\right)+10^{-4} \theta\left(|\omega|-v_{F} \Lambda\right)$. In addition, we concentrate on the case of the scalar disorder potential $U$. The results for a nonzero $\Delta$ will be discussed at the end of this section.

Let us first consider the case of a real potential shown in Fig. 1(a). As it is well known for graphene [57-62], the presence of impurities with a strong potential reshapes the LDOS and allows for the formation of resonance states for $|\omega|<v_{F} \Lambda$. With the increase of the potential strength, the corresponding peak moves to smaller frequencies and becomes sharper. In addition, there are peaks corresponding to the bound states at $|\omega|>v_{F} \Lambda$ that move to larger frequencies with an increase of $U$. (For demonstrative purposes we artificially broadened these peaks).

Further, we turn to one of the key results of this study related to imaginary and complex potentials. Unlike a real potential, a lossy one does not lead to the appearance of well-pronounced peaks in the LDOS [cf. Figs. 1(a) and 1(b)]. While, generically, it reduces the LDOS at large frequencies, it allows also for a weak enhancement at smaller ones. Moreover, the LDOS is no longer zero for $|\omega|>v_{F} \Lambda$. This is explained by the fact that an imaginary potential effectively interchanges real and imaginary parts in the denominator of the $T$ matrix. In the case of a complex potential, the peak in the DOS is broadened by a nonzero imaginary part of $U$ [see Fig. 1(c)]. In addition, due to the redistribution of the LDOS caused by $\operatorname{Im}[U] \neq 0$, the peak corresponding to the bound states becomes significantly broader.

Note that the case of attractive potential $\operatorname{Re}[U]<0$ is similar, albeit the resonance peaks appear at positive frequencies. In fact, the LDOS in this case can be obtained by reflecting $\omega \rightarrow-\omega$. Further, we note that since the linearized low-energy model is valid up to the energy cutoff $v_{F} \Lambda$, it is not able to reliably describe the formation of the bound states at $|\omega|>v_{F} \Lambda$. On the other hand, it correctly predicts their existence and evolution with the rise of the potential strength. 

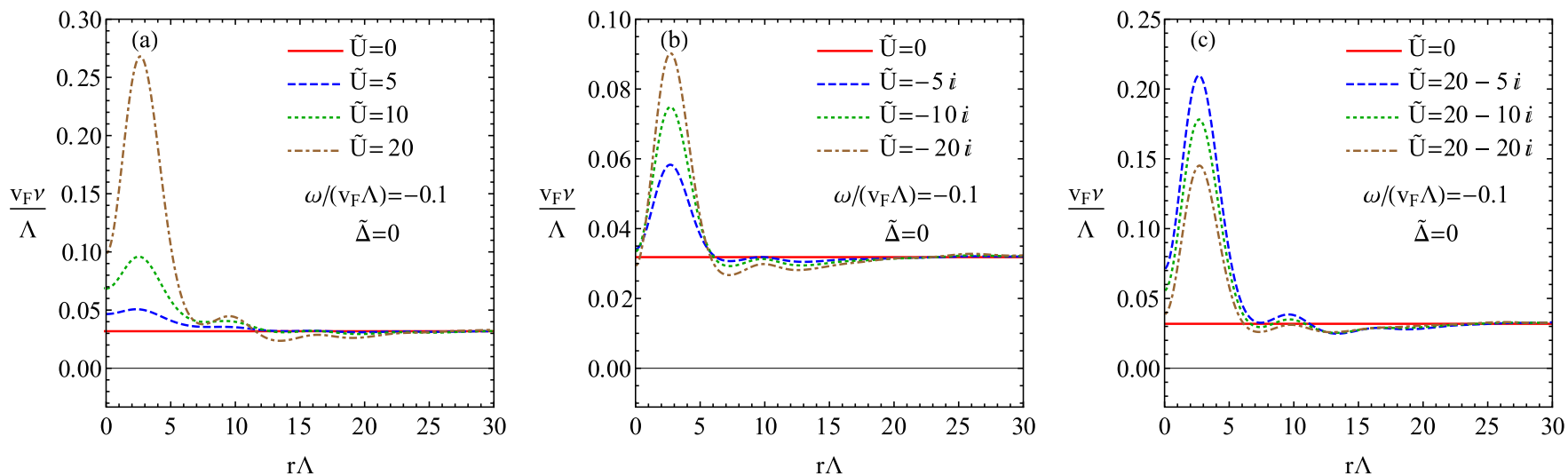

FIG. 2. The LDOS for real (a), imaginary (b), and complex (c) impurity potentials as a function of the distance from impurity $r$. Red solid, blue dashed, green dotted, and brown dot-dashed lines correspond to different disorder strengths shown in the corresponding legends. In addition, we set $\omega=-0.1 v_{F} \Lambda$ and $\tilde{U}=U \Lambda / v_{F}$ and $\Delta=0$.

A more rigorous description will be obtained in a lattice model in Sec. III.

Finally, let us discuss the spatial profiles of the LDOS. In view of the symmetry of the continuum model, we present only the dependence on the radial coordinate $r$. The corresponding results are shown in Figs. 2(a), 2(b), and 2(c) for a few values of real, imaginary (lossy), and complex potential at a fixed frequency, respectively. As one can see, the LDOS distribution has a characteristic profile with a dip at $r=0$ and a well-pronounced peak. Aside from the first peak, there are smaller side peaks, which are related to the Friedel oscillations. It is surprising that the purely imaginary dissipative potential also allows for a similar profile of the LDOS. The deviations of the LDOS due to the impurity are, however, much smaller. The peaks in the LDOS for a complex potential, where, in addition to a real part, there is a negative imaginary one, are suppressed for large $\operatorname{Im}[U]$. It is worth noting that since the linearized Dirac model does not work well for large wave vectors, the spatial dependence of the LDOS is not reliable at very small $r$. Nevertheless, as we will see in Sec. III, the enhancement of the LDOS in the vicinity of impurities persists also in a lattice model.
Before finalizing this section, let us briefly discuss the case of the impurity potential that intermixes valleys, i.e., $\Delta$. The results for the frequency dependence of the LDOS at the impurity site are shown in Fig. 3 at a fixed value of $\Delta \delta(\mathbf{r})$. As one can see from this figure, the local Dirac mass term leads to the impurity resonance peaks and bounds states on its own. Unlike the case of the impurity potential $U$, these peaks are symmetric with respect to the Dirac point. The presence of both types of the potentials enhances the LDOS for positive and negative frequencies. Furthermore, it makes peaks asymmetric, which, as we will see below, agrees with the results for a tight-binding model in Figs. 5 and 7. As for the spatial dependence of the LDOS, it remains qualitatively the same as for $U \neq 0$, where the LDOS reaches maximum near the impurity and oscillates far from it.

To summarize the key finding in this section, we show that the non-Hermitian dissipative impurity allows for a nontrivial frequency and spatial distribution of the LDOS. While the well-pronounced resonance peaks in frequency are absent, the spatial dependence of the LDOS is similar to the case of a usual impurity resonance. As is explicitly shown in
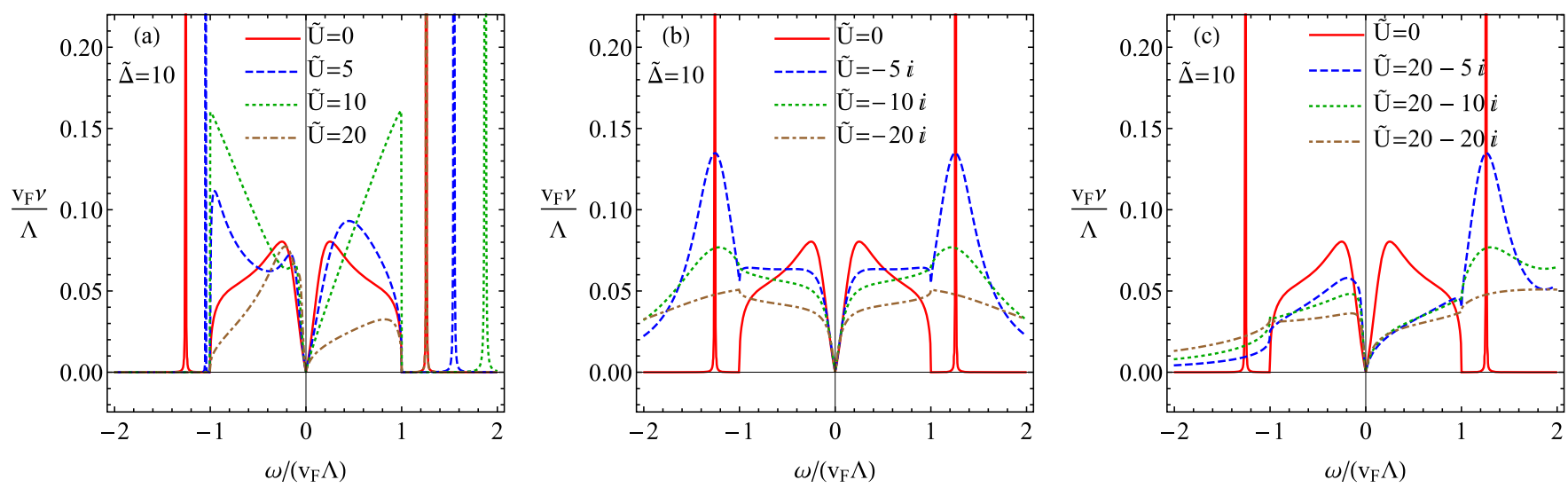

FIG. 3. The LDOS at the impurity site $v=v(\omega, \mathbf{0})$ for real (a), imaginary (b), and complex (c) impurity potentials as a function of the frequency $\omega$. The Dirac mass term at the impurity site is $\tilde{\Delta}=10$. Red solid, blue dashed, green dotted, and brown dot-dashed lines correspond to different disorder strengths shown in the corresponding legends. In addition, $\tilde{U}=U \Lambda / v_{F}$ and $\tilde{\Delta}=\Delta \Lambda / v_{F}$. 


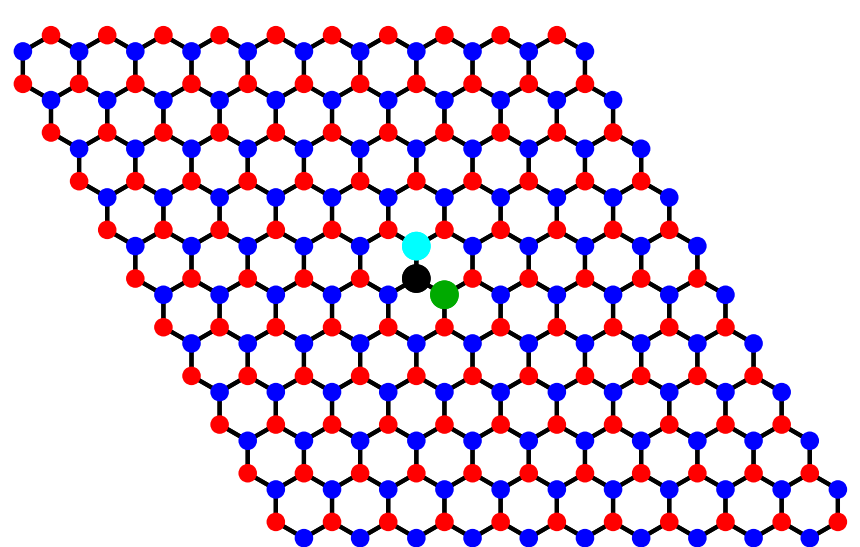

FIG. 4. Hexagonal lattice with $N=240$ sites $(10 \times 10$ hexagons) with zigzag boundary conditions. Red and blue dots corresponds to the $\mathrm{A}$ and $\mathrm{B}$ sublattices, respectively. The black and green dots mark impurity (defect) positions. The cyan dot is the neighboring site that will be used to present the frequency dependence of the LDOS.

Appendix A, the qualitative results found for the 2D model, remain valid also in $3 \mathrm{D}$.

\section{2D LATTICE MODEL}

\section{A. General definitions}

To investigate the effects related to the lattice symmetry and finite size as well as to support the results obtained in the continuum model, let us consider a tight-binding model for a 2D hexagonal lattice. Such a lattice qualitatively describes both real and photonic versions of graphene. Hexagonal lattice is defined by three vectors:

$$
\begin{aligned}
& \boldsymbol{\delta}_{1}=(0, a), \quad \boldsymbol{\delta}_{2}=\left(\frac{\sqrt{3} a}{2},-\frac{a}{2}\right), \\
& \boldsymbol{\delta}_{3}=\left(-\frac{\sqrt{3} a}{2},-\frac{a}{2}\right),
\end{aligned}
$$

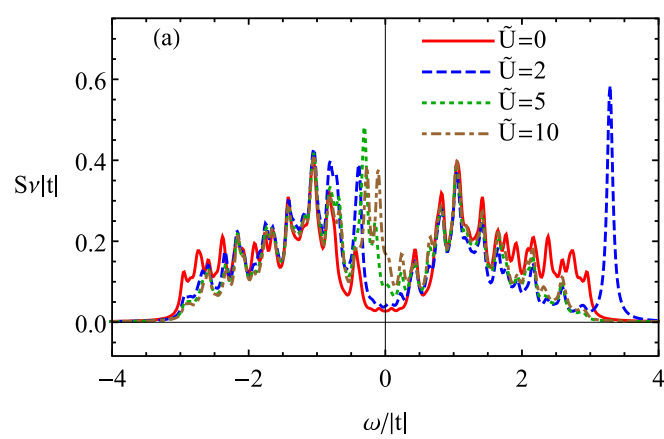

where $a$ is the lattice constant. The corresponding tightbinding Hamiltonian reads as

$$
\begin{aligned}
H= & -t \sum_{\mathbf{n}} \sum_{i=1}^{3}\left[\hat{a}_{\mathbf{n}}^{\dagger} \hat{b}_{\mathbf{n}+\delta_{i}}+\text { H.c. }\right] \\
& +U_{A} \sum_{\mathbf{l}} \hat{a}_{\mathbf{l}}^{\dagger} \hat{a}_{\mathbf{l}}+U_{B} \sum_{\mathbf{I}^{\prime}} \hat{b}_{\mathbf{I}^{\prime}}^{\dagger} \hat{b}_{\mathbf{I}^{\prime}},
\end{aligned}
$$

where $t$ is the hopping parameter, the creation (annihilation) operators $\hat{a}_{\mathbf{n}}^{\dagger}\left(\hat{a}_{\mathbf{n}}\right)$ and $\hat{b}_{\mathbf{n}}^{\dagger}\left(\hat{b}_{\mathbf{n}}\right)$ act on the sublattices $\mathrm{A}$ and $\mathrm{B}$, respectively, as well as $U_{A}$ and $U_{B}$ are the on-site impurity (defect) potentials. Further, the first sum runs over all lattice sites as well as the second and third sums accounts for defects on the A and B sublattices, respectively. We assume that there are $N$ lattice sites. As for the defects, the cases with one and two impurities will be discussed.

In what follows, we consider the case of a lattice with $N=240$ sites formed by $10 \times 10$ hexagons. We checked that the key results remain qualitatively the same for a small lattice with $N=48$ sites $(4 \times 4$ hexagons $)$ and for a larger one with $N=510$ sites $(15 \times 15$ hexagons $)$. In the former case, the distortions due to boundary effects are more noticeable, however. (The results for three lattice sizes are given in Appendix C.) The lattice together with the positions of the impurities is shown in Fig. 4. Note that we use the zigzag boundary conditions. The results should remain qualitatively the same for other types of the boundary conditions as long as the lattice is sufficiently large and the edges states do not interfere with the impurity resonance. To avoid the effects of boundaries as much as possible, the impurities are placed close to the center of the lattice. The positions of impurities are marked by the black and green dots in Fig. 4.

The LDOS for a lattice model is defined as

$$
\begin{aligned}
\nu\left(\omega, \mathbf{r}_{i}\right) & =\sum_{j} \psi_{j}^{\dagger}\left(\mathbf{r}_{i}\right) \psi_{j}\left(\mathbf{r}_{i}\right) \delta_{\tilde{\Gamma}}\left[\omega-\operatorname{Re}\left(\epsilon_{j}\right)\right] \\
& =\sum_{j} \frac{\psi_{j}^{\dagger}\left(\mathbf{r}_{i}\right) \psi_{j}\left(\mathbf{r}_{i}\right)}{\pi} \frac{\Gamma_{0}-\operatorname{Im}\left(\epsilon_{j}\right)}{\left[\omega-\operatorname{Re}\left(\epsilon_{j}\right)\right]^{2}+\left[\Gamma_{0}-\operatorname{Im}\left(\epsilon_{j}\right)\right]^{2}} .
\end{aligned}
$$

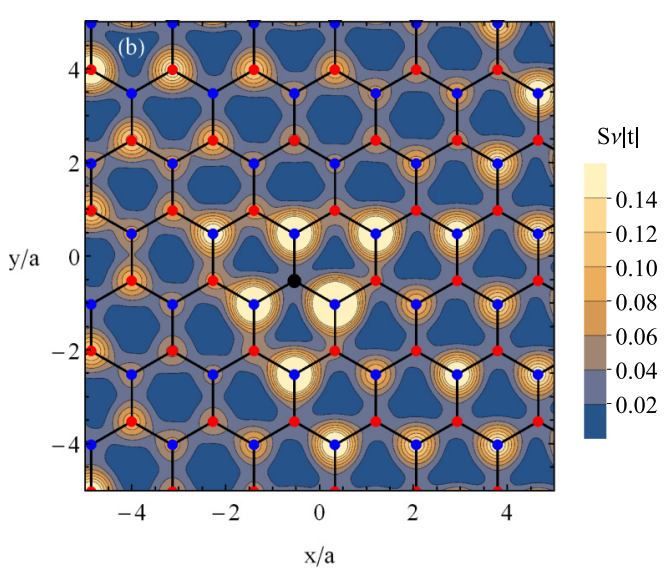

FIG. 5. (a) The LDOS at the neighboring site (see the cyan dot in Fig. 4) for real impurity potentials as a function of the frequency $\omega$. Red solid, blue dashed, green dotted, and brown dot-dashed lines correspond to different disorder strengths shown in the corresponding legend. (b) The spatial distribution of the LDOS for $\tilde{U}=10$ and $\omega=0$. The position of a single impurity is marked by the black dot. In both panels, $\tilde{U}=U /|t|$. 

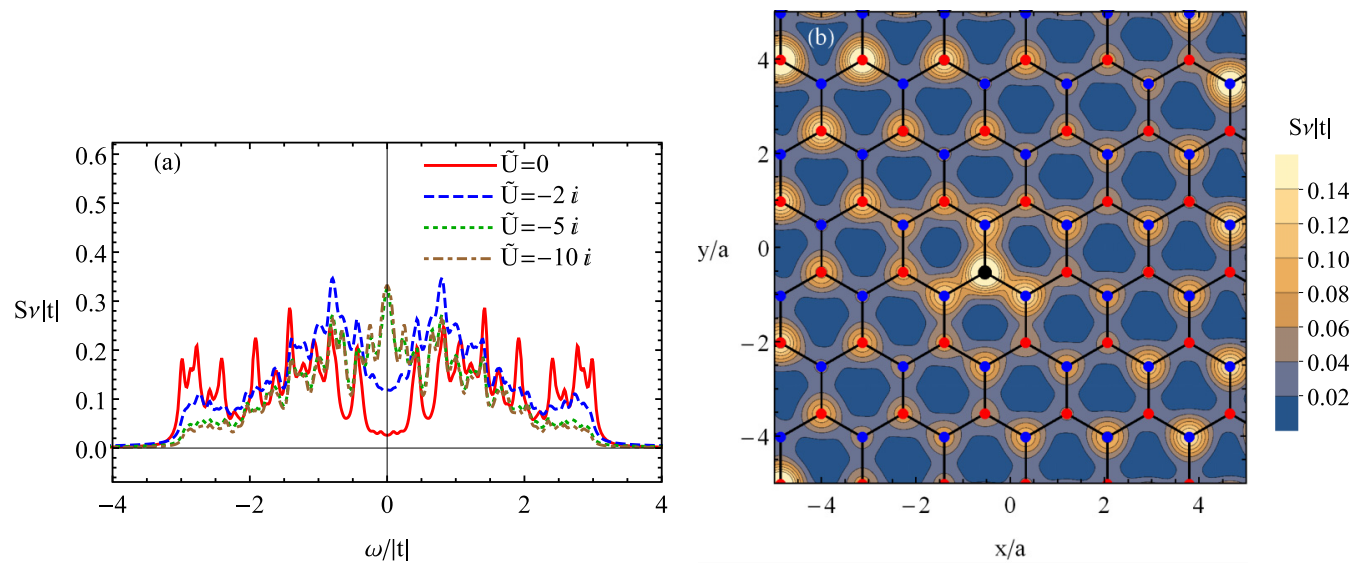

FIG. 6. (a) The LDOS at the neighboring site (see the cyan dot in Fig. 4) for imaginary impurity potentials as a function of the frequency $\omega$. Red solid, blue dashed, green dotted, and brown dot-dashed lines correspond to different disorder strengths shown in the corresponding legend. (b) The spatial distribution of the LDOS for $\tilde{U}=-2 i$ and $\omega=0$. The position of a single impurity is marked by the black dot. In both panels, $\tilde{U}=U /|t|$.

Here, $\mathbf{r}_{i}$ denotes the position of the site where the LDOS is calculated and $\sum_{j}$ runs over all eigenvalues $\epsilon_{j}$. Further, $\psi_{j}^{\dagger}\left(\mathbf{r}_{i}\right)$ is the normalized eigenvector corresponding to $\epsilon_{j}$ and $\tilde{\Gamma}=\Gamma_{0}-\operatorname{Im}\left(\epsilon_{j}\right)$. Note that we introduced a Lorentzian broadening of the $\delta$-function by $\Gamma_{0}$. This background broadening corresponds, e.g., to overall loss for a photonic lattice. The latter could be also modeled by adding on-site dissipative potential for all lattice sites. In our numerical simulations, it is assumed that $\Gamma_{0}=0.05|t|$. As we will demonstrate below, the presence of lossy impurities allows for $\operatorname{Im}\left[\epsilon_{j}\right]<0$, which will also contribute to the broadening.

As in the case of the continuum model, we consider the spatial distribution of the LDOS. In the model at hand, the LDOS is discrete and localized at lattice sites. Therefore, for presentation purposes, we introduce the spatial broadening by the normal distribution and define the following continuous LDOS:

$$
v(\omega, \mathbf{r})=\sum_{i} \frac{1}{\sqrt{2 \pi} r_{\mathrm{SD}}} e^{-\left(\mathbf{r}-\mathbf{r}_{i}\right)^{2} /\left(2 a^{2} r_{\mathrm{SD}}^{2}\right)} \nu\left(\omega, \mathbf{r}_{i}\right),
$$

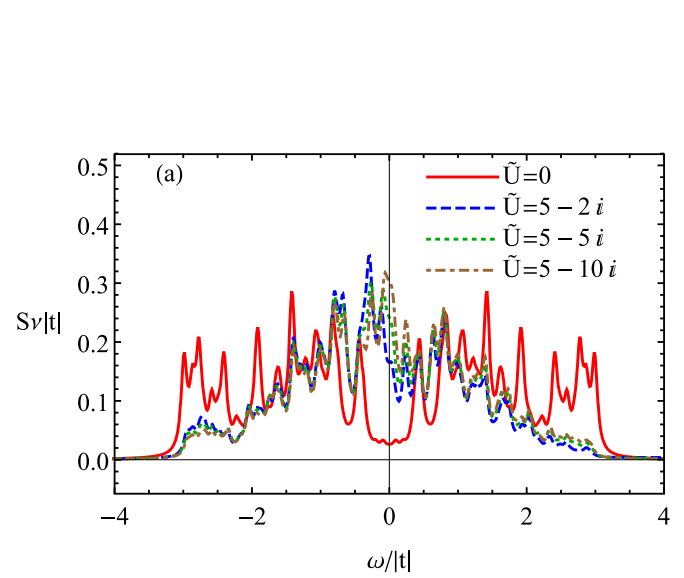

where $r_{\mathrm{SD}}$ is the standard deviation. We use $r_{\mathrm{SD}}=0.3$ in our numerical calculations.

\section{B. Hexagonal lattice with a single impurity}

In this section, we present the LDOS as a function of $\omega$ and coordinate for the lattice with a single impurity $U_{A}=U$ and $U_{B}=0$. The position of the latter is marked by the black dot in Fig. 4.

The dependence of the LDOS on $\omega$ at the neighboring site, which is marked by the cyan dot in Fig. 4, is shown in Figs. 5(a), 6(a), and 7(a) for real, lossy, and complex $U$, respectively. Here $S$ is the surface area of the lattice used to normalize the wave functions. Further, we present the spatial distribution of the LDOS at $\omega=0$ at a few values of the impurity potential in Figs. 5(b), 6(b), and 7(b).

Let us start with the case of real potentials. In agreement with the results for the continuum model (see Sec. II B) and previous studies [60-62], the real repulsive potential leads to the resonance peak in the LDOS at small $\omega$ [see Fig. 5(a)]

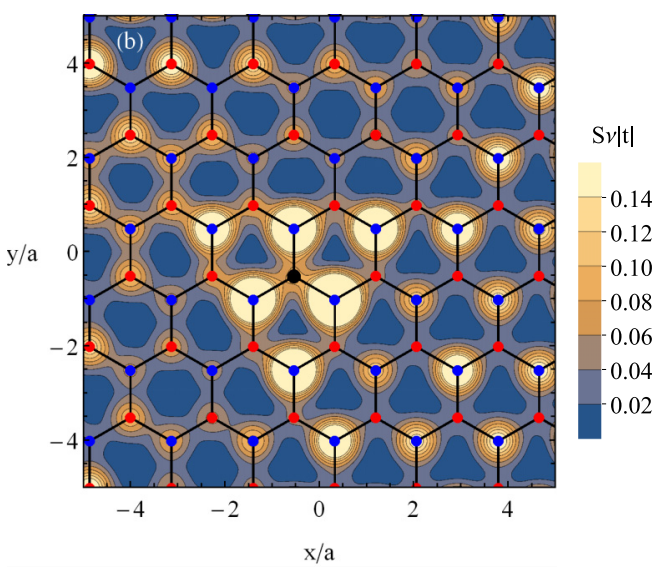

FIG. 7. (a) The LDOS at the neighboring site (see the cyan dot in Fig. 4) for complex impurity potentials as a function of the frequency $\omega$. Red solid, blue dashed, green dotted, and brown dot-dashed lines correspond to different disorder strengths shown in the corresponding legend. (b) The spatial distribution of the LDOS for $\tilde{U}=5-10 i$ and $\omega=0$. The position of a single impurity is marked by the black dot. In both panels, $\tilde{U}=U /|t|$. 

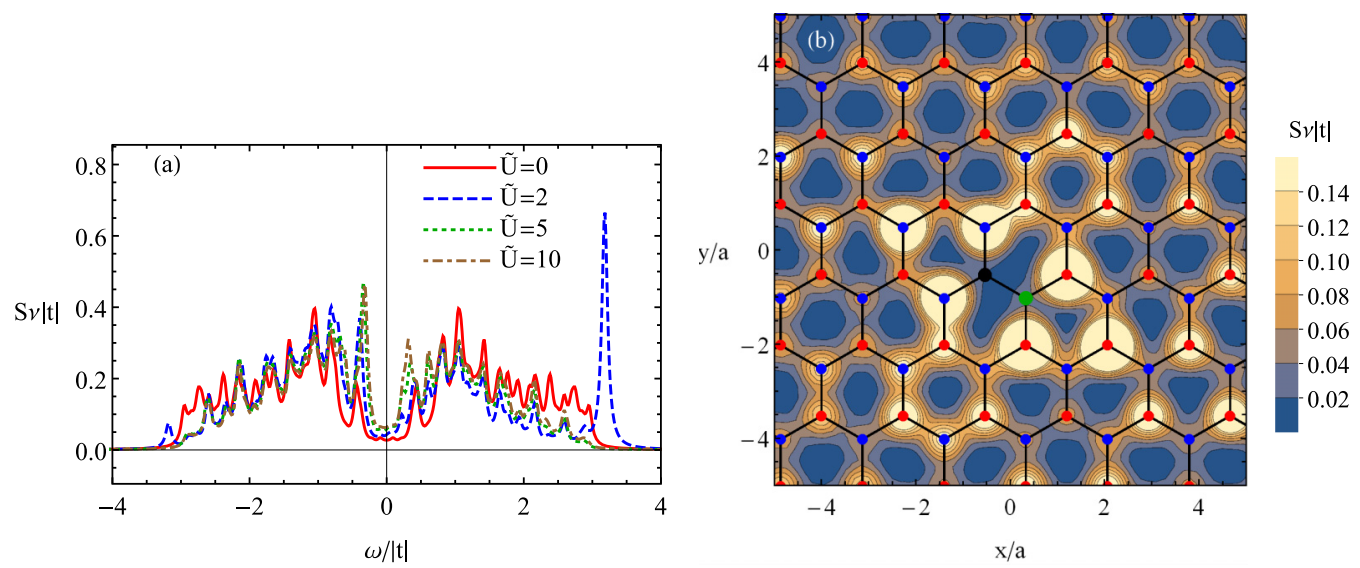

FIG. 8. (a) The LDOS at the neighboring site (see the cyan dot in Fig. 4) for real impurity potentials as a function of the frequency $\omega$. The values of the potential shown in the legend correspond to the site denoted by a black point. (b) The spatial distribution of the LDOS at $\omega=0.3|t|$ and $\tilde{U}=10$ for the impurity marked by the black dot. In both panels, $\tilde{U}=U /|t|$ and it is assumed that the potential at the other defect (green point) is the same in absolute value albeit has an opposite sign of the real part.

and allows for the formation of the trigonal-shaped LDOS structure in the vicinity of the impurity presented in Fig. 5(b). The lattice results suggest that the LDOS at the impurity site itself should be much lower than its neighbourhood. The peaks in Fig. 5(a) flatten with increasing the lattice size and performing averaging over the neighboring sites. Note also that the LDOS is accumulated primarily at B-sublattice sites if the defect is on an A sublattice. In addition, as in the case of continuum model, the bound state occurs for larger frequencies [see the peak in Fig. 5(a) at $\tilde{U}=2$ ]. With the rise of impurity potential, it moves to larger frequencies and its width decreases.

The presence of the purely imaginary potential also allows for additional features in the LDOS. In particular, as one can see from Fig. 6(a), the LDOS at small $\omega$ is enhanced and even forms a peak at $\omega=0$ for a strong potential. While this feature is absent for the continuum model, it originates from the fact that the DOS is nonzero due to finite size effects. As for the spatial distribution of the LDOS, it is enhanced at the impurity site at relatively small values of $\operatorname{Im}[U]$, albeit acquires the trigonal shape similar to that in Fig. 5(b) when $\operatorname{Im}[U]$ becomes large. For a detailed discussion on the corresponding dependence of the LDOS, see Appendix B. Finally, the results in Fig. 7 show that the imaginary part of the impurity potential could enhance the resonance peak for a complex potential but does not modify much the spatial pattern of the LDOS and the position of the peaks.

Thus, in agreement with our finding for the continuum model, the results for the hexagonal lattice also demonstrate the appearance of the distinctive signature in the LDOS for a dissipative potential. Depending on the magnitude of the potential, one can observe an enhancement of the LDOS at the impurity site or the conventional trigonal patter of the LDOS. In addition, the results for a few different lattice sizes presented in Appendix $\mathrm{C}$ demonstrate that the impurity resonance persists both in small $(4 \times 4$ hexagons $)$ and large $(15 \times 15$ hexagons $)$ lattices.
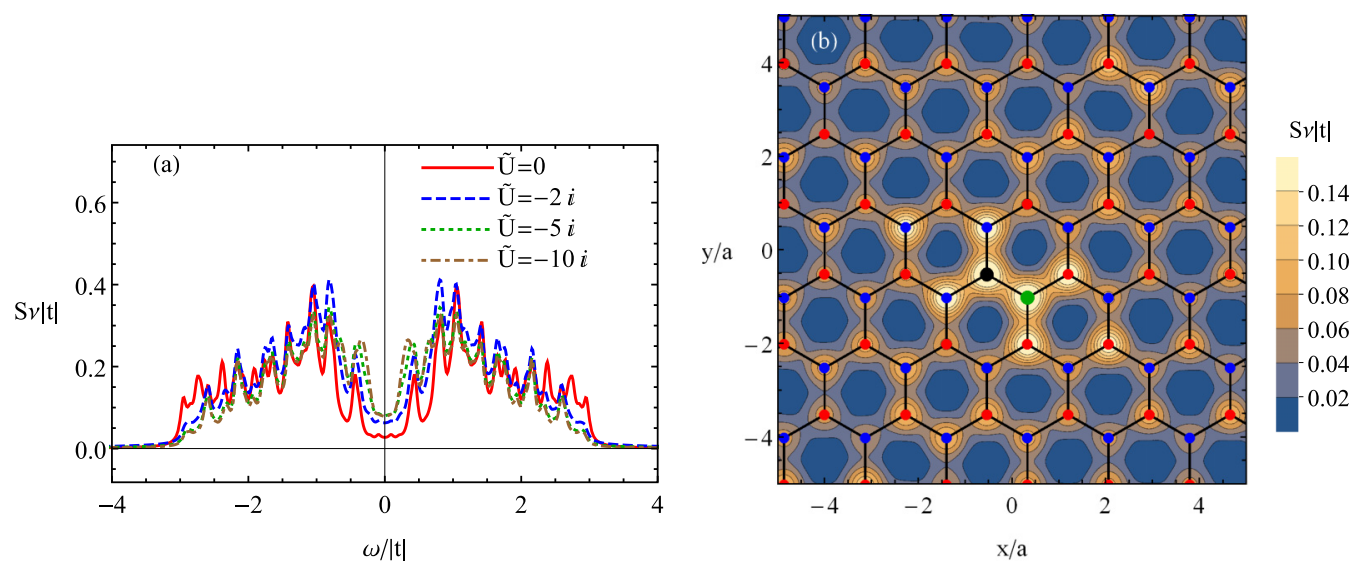

FIG. 9. (a) The LDOS at the neighboring site (see the cyan dot in Fig. 4) for imaginary impurity potentials as a function of the frequency $\omega$. The values of the potential shown in the legend correspond to the site denoted by the black point. (b) The spatial distribution of the LDOS at $\omega=0.3|t|$ and $\tilde{U}=-2 i$ for the impurity marked by the black dot. In both panels, $\tilde{U}=U /|t|$ and it is assumed that the potential at the other defect (green point) is the same in absolute value albeit has an opposite sign of the real part. 


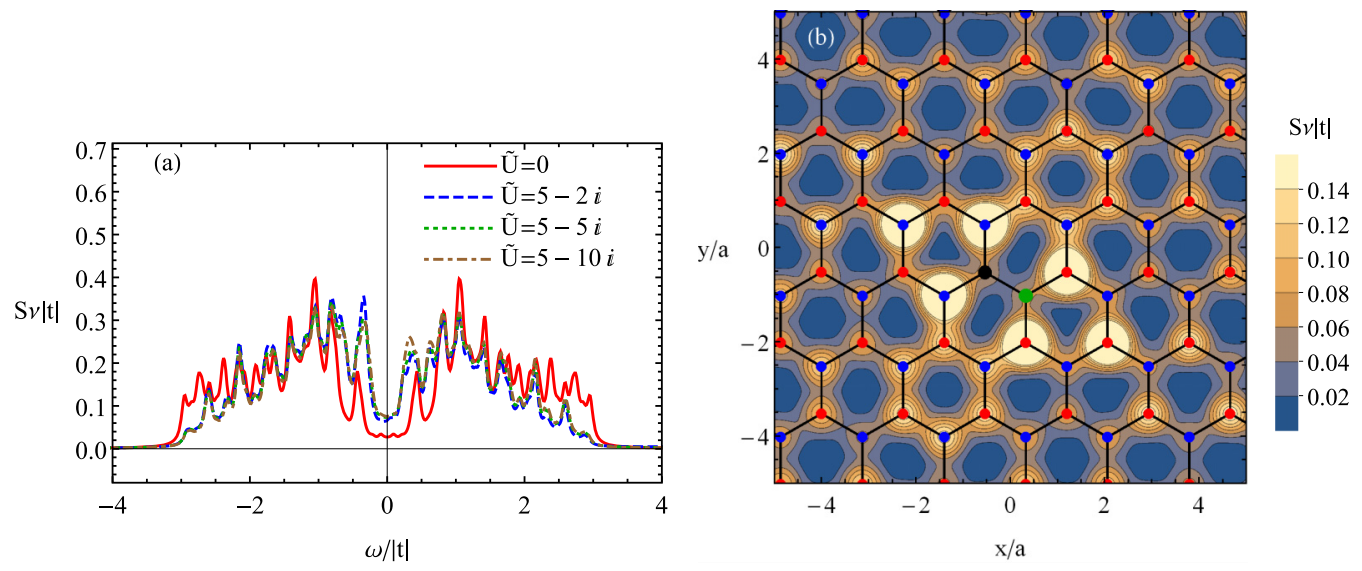

FIG. 10. (a) The LDOS at the neighboring site (see the cyan dot in Fig. 4) for complex impurity potentials as a function of the frequency $\omega$. The values of the potential shown in the legend correspond to the site denoted by the black point. (b) The spatial distribution of the LDOS at $\omega=0.3|t|$ and $\tilde{U}=5-10 i$ for the impurity marked by the black dot. In both panels, $\tilde{U}=U /|t|$ and it is assumed that the potential at the other defect (green point) is the same in absolute value albeit has an opposite sign of the real part.

\section{Hexagonal lattice with two impurities}

In this section, we consider the case of two impurities placed on the neighboring sites. Their position is marked by the black and green dots in Fig. 4. For the sake of brevity, let us investigate only the most interesting case in which the sign of the real part of the impurity potential is opposite for two defects, i.e., $\operatorname{Re}\left[U_{A}\right]=-\operatorname{Re}\left[U_{B}\right]=\operatorname{Re}[U]$ and $\operatorname{Im}\left[U_{A}\right]=$ $\operatorname{Im}\left[U_{B}\right]=\operatorname{Im}[U]$. In particular, this corresponds to a dipolelike impurity where one of the sites is repulsive $(\operatorname{Re}[U]>0)$ and the other is attractive $(\operatorname{Re}[U]<0)$. The corresponding results for the LDOS as a function of $\omega$ and coordinates are shown in Figs. 8, 9, and 10 for real, imaginary, and complex potentials, respectively.

It is interesting that while the resonance peaks do appear in the LDOS for real impurity potentials, the LDOS at $\omega \rightarrow 0$ is only trivially shifted [see Fig. 8(a)] but becomes asymmetric at $\omega \neq 0$. Therefore, in order to probe the nontrivial distribution of the LDOS, a small but nonzero frequency should be used. In particular, we obtained a double-trigonal structure of the LDOS shown in Fig. 8(b) at $\omega=0.3|t|$. Note also that the LDOS is accumulated at B-sublattice (A-sublattice) sites near the impurity placed at the A-type (B-type) site.

As in the case of a single impurity, the purely dissipative impurity potentials for two impurities also affects the LDOS. As one can see from Fig. 9(a), the LDOS at $\omega \rightarrow 0$ is enhanced. As in the case of a single impurity, the shape of the spatial profile of the LDOS depends on the magnitude of the potential. For a relatively small $|U| \sim|t|$, the LDOS in enhanced on the impurity sites. This LDOS quickly acquires double-trigonal shape for large $|U|$, however. (For the details of the LDOS evolution, see Appendix B.) The results for the dipole potential with a nonzero dissipative part are shown in Figs. 9 and 10. There is an enhancement of the LDOS at small $\omega$ due to the presence of the imaginary part of the potential as well as asymmetry related to $\operatorname{Re}[U]$.

\section{CONCLUSIONS}

In this study, we investigated the effects of non-Hermitian defects on the properties of Dirac systems. By using con- tinuum and lattice models, we found that, similarly to conventional impurities described by real potentials, the purely imaginary lossy defects also allow for a nontrivial spatial distribution of the LDOS. The latter is particularly interesting since the corresponding potential does not lead to noticeable peaks in the frequency profile of the DOS.

In agreement with the previous literature, we found that the resonance peaks occur in the frequency profile of the DOS for a real impurity potential in a continuum model of 2D and 3D Dirac materials. The position of these peaks is controlled by the potential strength $|U|$. In particular, the peaks move to smaller frequencies with the rise of $|U|$. The impurity resonance is manifested also in the spatial profile of the LDOS, where a noticeable peak occurs in the vicinity of the impurity.

We obtained that a similar profile of the LDOS appears also for a purely lossy potential. The dependence on the frequency is, however, different. Instead of well-pronounced peaks, the LDOS is only slightly enhanced at small frequencies and diminishes for larger ones. In addition, the LDOS becomes nonzero even for frequencies above the cutoff. In the case of complex impurity potentials with both real and imaginary parts, the enhancement of the LDOS above the cutoff broadens the peaks of bound states at large frequencies, which could make their observation easier. The corresponding signal could be, however, hindered due to possible overlap with other bands. A rigorous description of the bound states requires an approach more refined that the linearized model.

The numerical results for the 2D hexagonal lattice model with a single impurity support the analytical analysis. In particular, the resonance peaks in the LDOS on a neighboring site appear for a real impurity potential at small frequencies. Moreover, the LDOS is enhanced at $\omega=0$ for a purely lossy defect and an impurity with a complex potential. In all these cases, the presence of a defect is manifested in the characteristic trigonal-shaped LDOS around the impurity when the potential is sufficiently strong. The observation of such a distribution of the LDOS for a lossy site would be a definitive signature of the non-Hermitian defect state. 

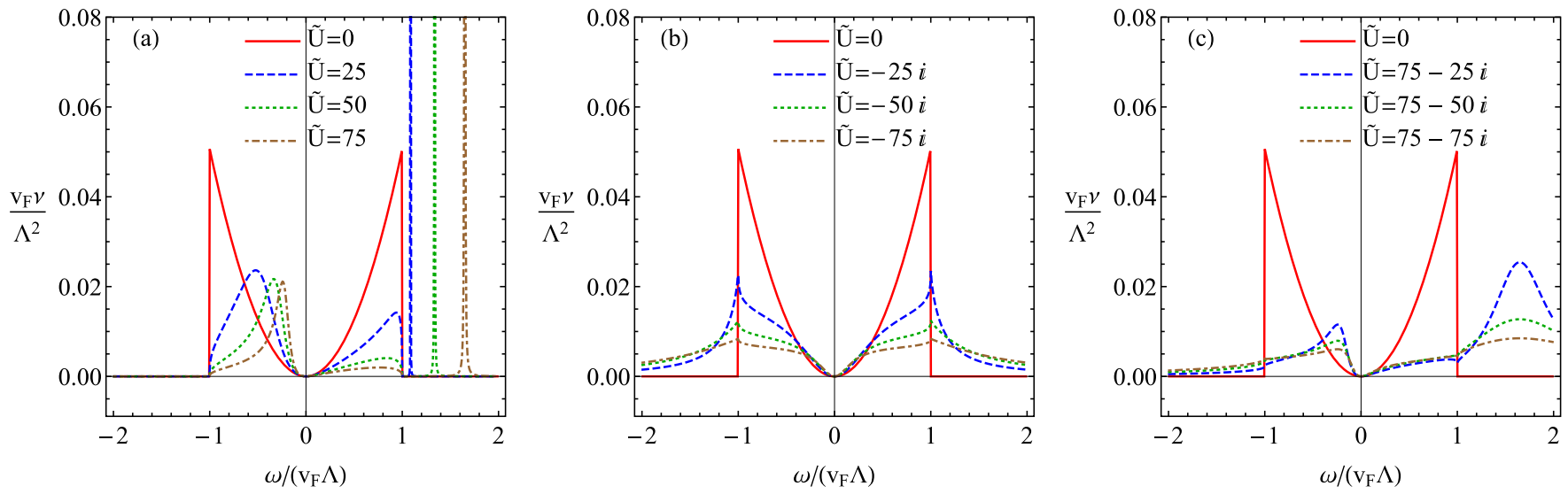

FIG. 11. The LDOS at the impurity site for real (a), imaginary (b), and complex (c) impurity potentials as a function of the frequency $\omega$. Red solid, blue dashed, green dotted, and brown dot-dashed lines correspond to different disorder strengths shown in the corresponding legends. In addition, $\tilde{U}=U \Lambda^{2} / v_{F}$.

In the case of a dipole impurity, where the real parts of the defect potentials have opposite signs on the neighboring defects, the LDOS as a function of frequency as well as the spatial distribution of the LDOS qualitatively differ from the case of a single defect. In particular, while a resonance peak occurs at small frequencies for a real potential, the LDOS at $\omega=0$ is only trivially shifted. The same is also valid for imaginary and complex potential. It is found that the spatial distributions of the LDOS for real, imaginary, and complex impurity potentials have a characteristic double-trigonal form for large $|U|$. In addition, we checked that the qualitative features related to the defect states persist also for smaller lattices, where, however, the effects of boundaries become important.

Let us also briefly discuss the experimental feasibility of our setup. Defects of arbitrary configuration can be straightforwardly realized on-demand in photonic lattices. For example, the impurity potential is directly determined by the size of waveguides. A lossy potential could be achieved by introducing disorder inside the waveguide and/or by not writing it completely (i.e., by leaving regions in the waveguide filled with a background material). Thus, we believe that the model setup of this study can be straightforwardly realized in optical experiments by using a hexagonal photonic lattice. We can speculate that the lossy potential could be also modeled in solids by introducing a sink for electrons (e.g., by employing a point contact).

\section{ACKNOWLEDGMENTS}

We are grateful to M. Bourennane, W. Cherifi, and S. Bandyopadhyay for useful discussions. This work was supported by the VILLUM FONDEN via the Centre of Excellence for Dirac Materials (Grant No. 11744), the European Research Council under the European Unions Seventh Framework Program Synergy HERO, and the Knut and Alice Wallenberg Foundation KAW 2018.0104.

\section{APPENDIX A: 3D CONTINUUM DIRAC MODEL}

In this section, we consider impurity effects in the three-dimensional (3D) continuum model. The corresponding Hamiltonian is given in Eq. (1) in the main text. By using the $T$-matrix approach described in Sec. II A, the resonance and bound states can be identified. In particular, they are given by the poles of the $T$ matrix. In the case of 3D Dirac Hamiltonian, the corresponding characteristic equation that determines the position of the resonances reads as

$$
0=1-\operatorname{Re} \sum_{\mathbf{k}} U \gamma^{0} G_{0}^{\mathrm{R}}(\omega, \mathbf{k})
$$

where

$$
\begin{aligned}
\sum_{\mathbf{k}} U \gamma^{0} G_{0}^{\mathrm{R}}(\omega, \mathbf{k}) & =\int \frac{d^{3} k}{(2 \pi)^{3}} U \gamma^{0} G_{0}^{\mathrm{R}}(\omega, \mathbf{k})=U \int \frac{d^{3} k}{(2 \pi)^{3}} \frac{\omega-v_{F} \gamma^{0}(\boldsymbol{\gamma} \cdot \mathbf{k})}{\omega^{2}-v_{F}^{2} k^{2}+i 0 \operatorname{sgn}(\omega)} \\
& =U \frac{\omega}{2 \pi^{2}} \int_{0}^{\Lambda} d k k^{2}\left[\text { v.p. } \frac{1}{\omega^{2}-v_{F}^{2} k^{2}}-i \pi \operatorname{sgn}(\omega) \delta\left(\omega^{2}-v_{F}^{2} k^{2}\right)\right] \\
& =U \frac{1}{2 \pi^{2} v_{F}^{3}}\left[-v_{F} \Lambda \omega+\frac{\omega^{2}}{2} \ln \left|\frac{\omega+v_{F} \Lambda}{\omega-v_{F} \Lambda}\right|-i \frac{\pi}{2} \omega^{2} \theta\left(v_{F}^{2} \Lambda^{2}-\omega^{2}\right)\right] .
\end{aligned}
$$

Here, $U$ is the impurity potential (for simplicity we ignored the chirality-mixing potential $\sim \Delta), G_{0}^{\mathrm{R}}(\omega, \mathbf{k})$ is the bare re- tarded Green's function given in Eq. (3), $\Lambda$ is the momentum cutoff, $v_{F}$ is the Fermi velocity, v.p. stands for the principal 

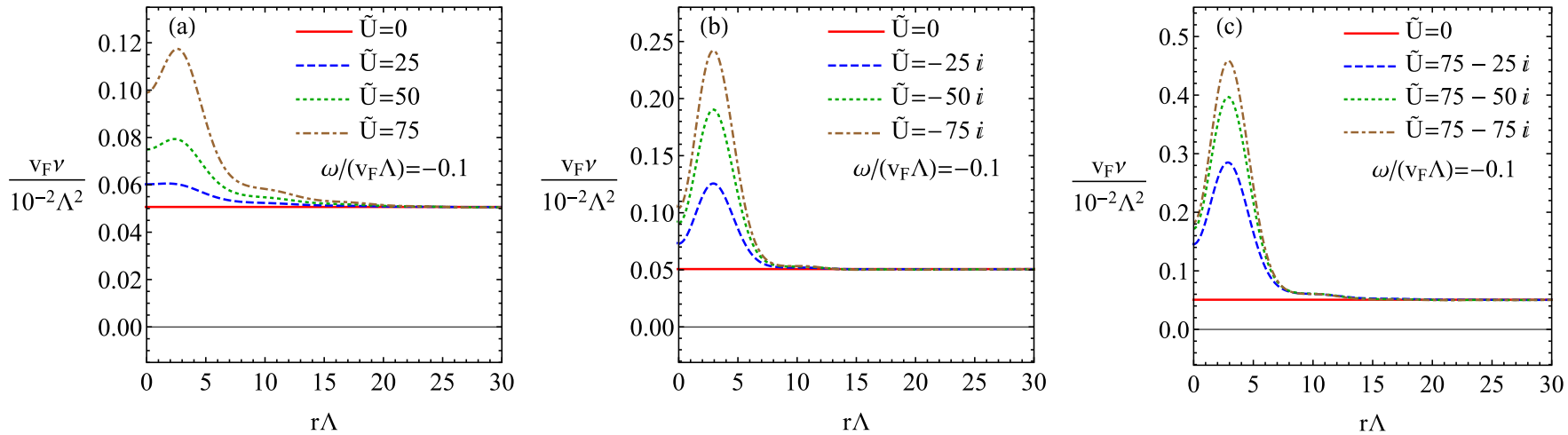

FIG. 12. The LDOS for real (a), imaginary (b), and complex (c) impurity potentials as a function of the distance to impurity $r$. Red solid, blue dashed, green dotted, and brown dot-dashed lines correspond to different disorder strengths shown in the corresponding legends. In addition, we used $\omega=-0.1 v_{F} \Lambda$ and $\tilde{U}=U /\left(v_{F} \Lambda\right)$.

value, and $\theta(x)$ is the step function. The key difference from the two-dimensional (2D) case considered in Sec. II B is in the overall scaling of the integrated Green's function with $\omega$. Indeed, as one can see from the results for the on-site LDOS in Fig. 11(a), the impurity resonance peaks have a similar form albeit appear on top of the parabolic density of states (DOS) defined for a single Weyl node as

$$
v_{0}=\frac{\omega^{2}}{2 \pi^{2} v_{F}^{3}} \theta\left(v_{F} \Lambda-|\omega|\right) .
$$
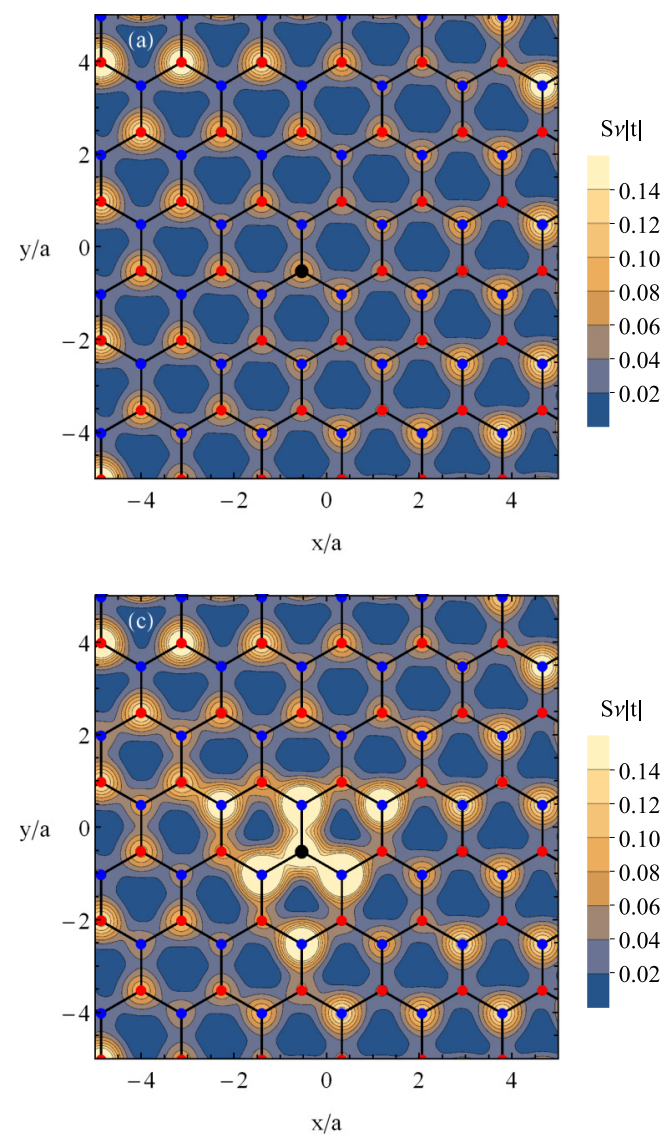

Further, as follows from Figs. 11(b) and 11(c), the LDOS at the impurity site becomes nonzero for $|\omega|>v_{F} \Lambda$ when $\operatorname{Im}[U] \neq 0$. Therefore the key features of the impurity resonances and the non-Hermitian defect states resemble those for 2D Dirac systems considered in Sec. II B.

As for the spatial profiles of the LDOS, which are shown in Fig. 12, the results are also similar to those in the $2 \mathrm{D}$ case. The form of the peaks is, however, slightly different. In particular, while the LDOS for real potentials has a less-pronounced peak nearby the impurity [cf. Figs. 2(a) and 12(a)], the peak is more noticeable for a dissipative $U$. In addition, while
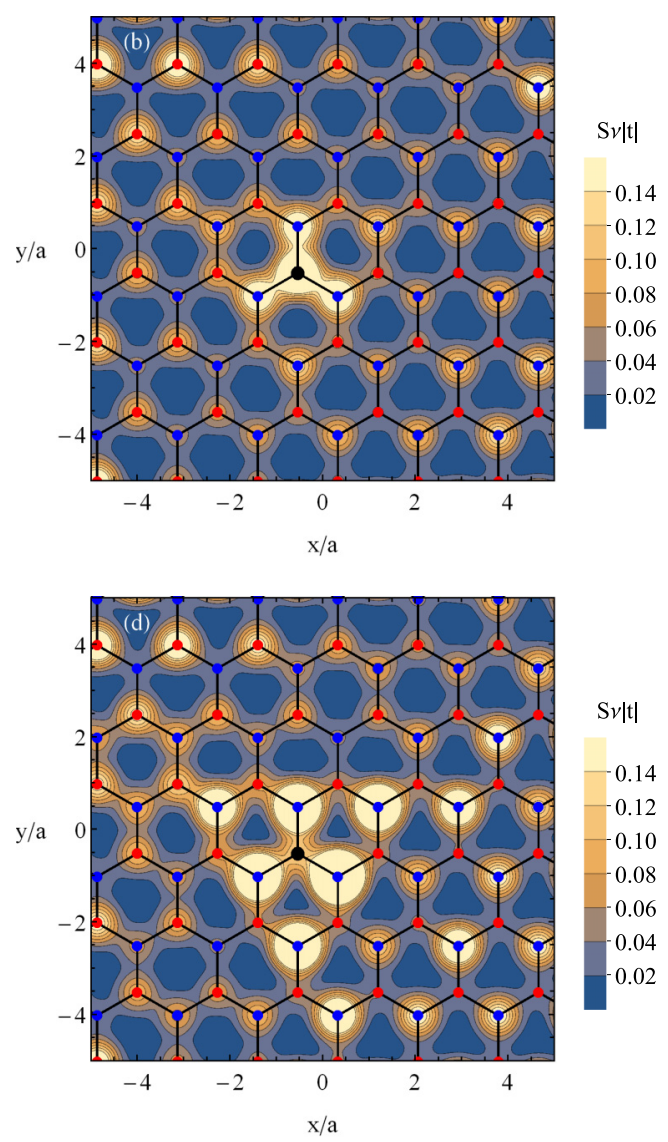

FIG. 13. The spatial distribution of the LDOS for $\omega=0$. The position of a single impurity is marked by the black dot. We set $U=-i|t|$ (a), $U=-3 i|t|$ (b), $U=-5 i|t|$ (c), and $U=-10 i|t|$ (d). 

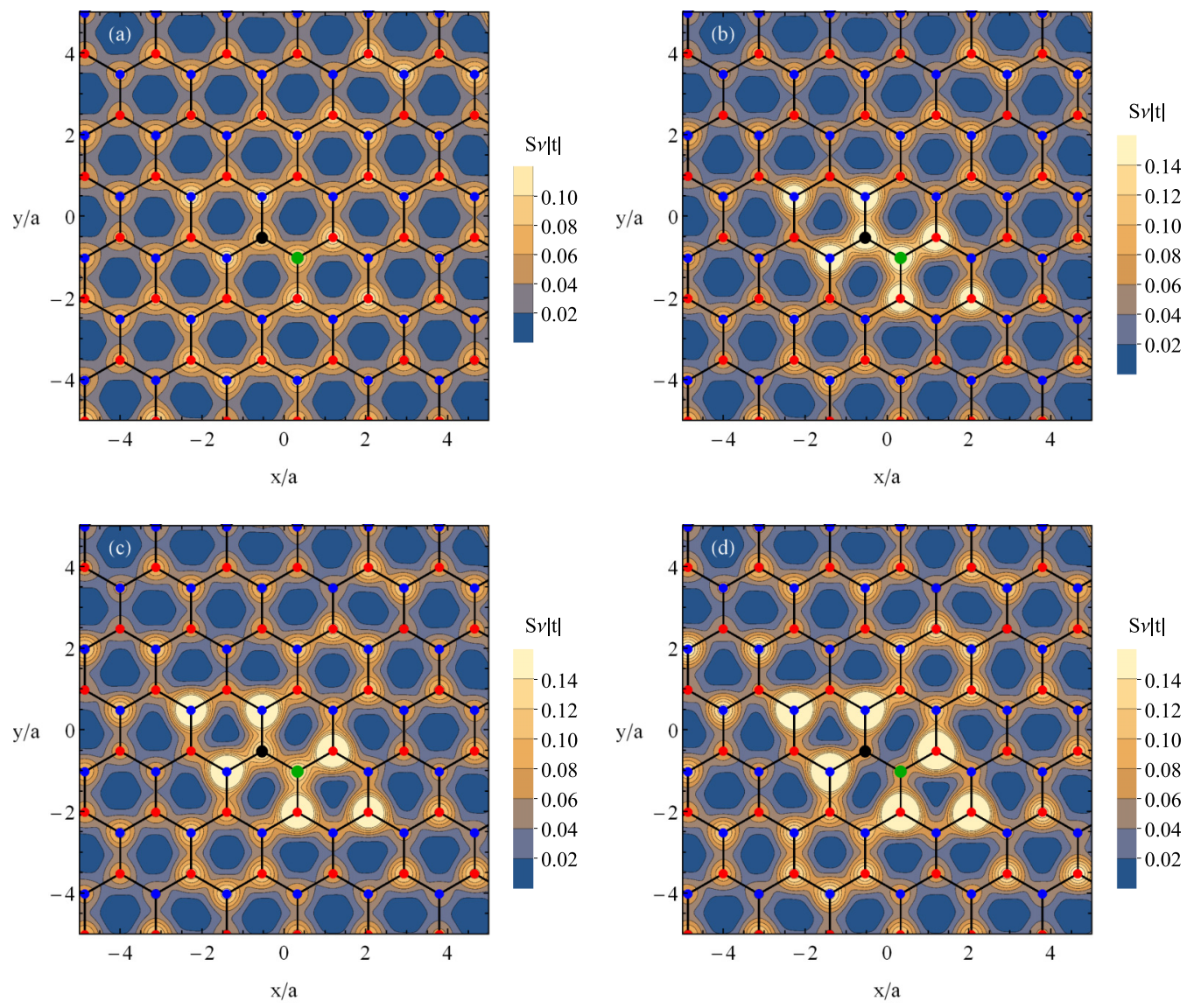

FIG. 14. The spatial distribution of the LDOS for $\omega=0.3|t|$. The position of two impurities is marked by the black and green dots. We set $U=-i|t|$ (a), $U=-3 i|t|(\mathrm{b}), U=-5 i|t|$ (c), and $U=-10 i|t|(\mathrm{d})$.

the magnitude of the peak is reduced by an imaginary part of the complex impurity potential in $2 \mathrm{D}$, the situation is opposite in the $3 \mathrm{D}$ case. It is worth noting also that while the LDOS in 2D materials is directly accessible to scanning tunneling microscopy probes, it might be hard to investigate bulk impurity resonances in 3D.

\section{APPENDIX B: LDOS FOR PURELY IMAGINARY DEFECTS IN HEXAGONAL LATTICE MODEL}

In this section, we demonstrate the evolution of the LDOS in the hexagonal lattice model described in Sec. III A. In particular, we concentrate on the case of a purely imaginary disorder with $\operatorname{Re}\left[U_{A}\right]=\operatorname{Re}\left[U_{B}\right]=\operatorname{Re}[U]=0$ and a negative imaginary part of the potential, where the dependence of the LDOS on the potential strength $U$ is nonmonotonic. The results for a single impurity $\operatorname{Im}\left[U_{A}\right]=\operatorname{Im}[U]$ and $\operatorname{Im}\left[U_{B}\right]=0$ placed at the site marked by the black dot in Fig. 4 are shown in a few panels of Fig. 13. As one can see, the LDOS first increases at the impurity site [see Figs. 13(a) and 13(b)]. Then, with the rise of the absolute value of the potential, the trigonal pattern in the LDOS starts to manifest [see Figs. 13(c) and 13(d)]. The LDOS in the latter case is similar to that for real defect potential shown in Fig. 5(b).
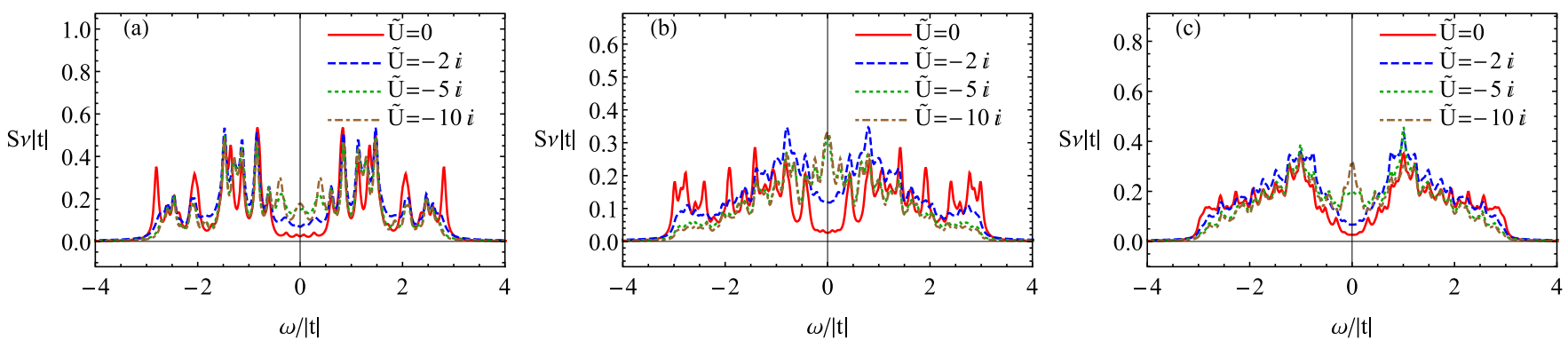

FIG. 15. The frequency dependence of the LDOS at the neighboring site (see the cyan dot in Fig. 4) for $\tilde{U}=0$ (red solid line), $\tilde{U}=-2 i$ (blue dashed line), $\tilde{U}=-5 i$ (green dotted line), and $\tilde{U}=-10 i$ (brown dot-dashed line). We consider the systems with $4 \times 4$ (a), $10 \times 10$ (b), and $15 \times 15$ (c) hexagons. Here, $\tilde{U}=U /|t|$. 

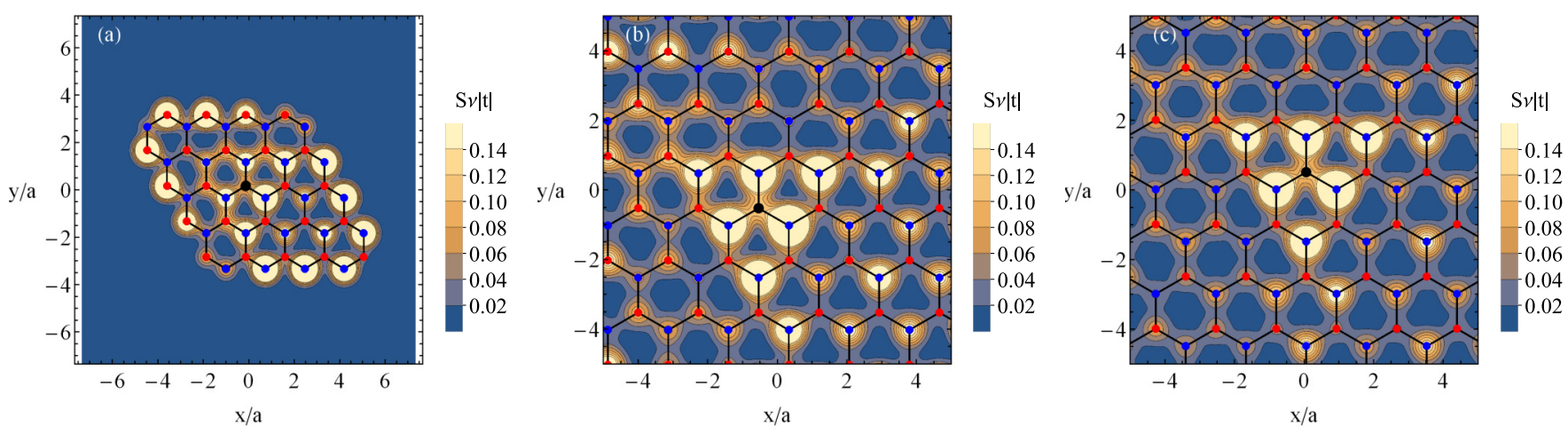

FIG. 16. The spatial distribution of the LDOS for $\tilde{U}=-10 i$. We consider the systems with $4 \times 4$ (a), $10 \times 10$ (b), and $15 \times 15$ (c) hexagons. Here, $\tilde{U}=U /|t|$.

The results for two defects with the same imaginary potential $\operatorname{Im}\left[U_{A}\right]=\operatorname{Im}\left[U_{B}\right]=\operatorname{Im}[U]$ (see the black and green dots in Fig. 4) are shown in Fig. 14. Similarly to the case of a single defect considered before, the dependence of the LDOS on the impurity strength is nonmonotonic. While it reaches maximum on the defect sites for small values of impurity potential, the double-trigonal pattern appears for large $|U|$.

\section{APPENDIX C: LDOS DEPENDENCE ON LATTICE SIZE}

To demonstrate the evolution of the LDOS with the lattice size, we consider an impurity with a purely imaginary potential in a few lattices. In particular, a small lattice with $4 \times 4$ hexagons as well as two large lattices with $10 \times 10$ and $15 \times 15$ hexagons are discussed. The frequency dependence of the LDOS near the impurity site is shown in three panels of Fig. 15 for a few values of the imaginary potential. The spatial distributions of the LDOS near the defect are shown in three panels of Fig. 16 at $\tilde{U}=-10 i$.

We found that the qualitative features of the impurity defect states remain the same regardless of the size of the system. On the other hand, there are a few quantitative differences. In particular, the peaks in the frequency dependence of the LDOS are much sharper in the case of a small lattice and gradually smoothen for a larger one [cf. Fig. 15(a) with Figs. 15(b) and 15(c)]. Further, we notice that the shape of the resonance states slightly deviates from the trigonal one for a very small lattice [see Fig. 16(a)]. In addition, the effects of the edge states become more pronounced in this case. On the other hand, the results for larger lattices [see Figs. 16(b) and 16(c)] are very similar and suggest that the trigonal profile of the LDOS around the impurity indeed corresponds to the impurity resonance rather than is a finite-size effect.
[1] N. Moiseyev, Non-Hermitian Quantum Mechanics (Cambridge University Press, Cambridge, 2011).

[2] C. M. Bender, PT Symmetry: In Quantum and Classical Physics (World Scientific, Singapore, 2018).

[3] C. M. Bender, Contemp. Phys. 46, 277 (2005).

[4] C. M. Bender, Rep. Prog. Phys. 70, 947 (2007).

[5] A. Mostafazadeh, Int. J. Geom. Methods Mod. Phys. 07, 1191 (2010).

[6] K. G. Makris, R. El-Ganainy, D. N. Christodoulides, and Z. H. Musslimani, Int. J. Theor. Phys. 50, 1019 (2011).

[7] V. V. Konotop, J. Yang, and D. A. Zezyulin, Rev. Mod. Phys. 88, 035002 (2016).

[8] C. M. Bender and S. Boettcher, Phys. Rev. Lett. 80, 5243 (1998).

[9] C. M. Bender, S. Boettcher, and P. N. Meisinger, J. Math. Phys. 40, 2201 (1999).

[10] R. El-Ganainy, M. Khajavikhan, D. N. Christodoulides, and S. K. Ozdemir, Commun. Phys. 2, 37 (2019).

[11] K. Kawabata, S. Higashikawa, Z. Gong, Y. Ashida, and M. Ueda, Nat. Commun. 10, 297 (2019).

[12] K. Kawabata, K. Shiozaki, M. Ueda, and M. Sato, Phys. Rev. X 9, 041015 (2019).

[13] H. Zhou and J. Y. Lee, Phys. Rev. B 99, 235112 (2019).
[14] Y. Ota, K. Takaka, T. Ozawa, A. Amo, Z. Jia, B. Kante, M. Notomi, Y. Arakawa, and S. Iwamoto, Nanophotonics 9, 547 (2020).

[15] T. Yoshida, K. Kudo, and Y. Hatsugai, Sci. Rep. 9, 16895 (2019).

[16] T. Yoshida, R. Peters, N. Kawakami, and Y. Hatsugai, Phys. Rev. B 99, 121101(R) (2019).

[17] L. E. F. Foa Torres, J. Phys.: Mater. 3, 014002 (2020).

[18] S. Yao and Z. Wang, Phys. Rev. Lett. 121, 086803 (2018).

[19] S. Yao, F. Song, and Z. Wang, Phys. Rev. Lett. 121, 136802 (2018).

[20] V. M. Martinez Alvarez, J. E. Barrios Vargas, M. Berdakin, and L. E. F. Foa Torres, Eur. Phys. J.: Spec. Top. 227, 1295 (2018).

[21] Y. Xiong, J. Phys. Commun. 2, 035043 (2018).

[22] C. H. Lee and R. Thomale, Phys. Rev. B 99, 201103(R) (2019).

[23] D. S. Borgnia, A. J. Kruchkov, and R.-J. Slager, Phys. Rev. Lett. 124, 056802 (2020)

[24] H.-G. Zirnstein, G. Refael, and B. Rosenow, arXiv:1901.11241.

[25] T. Yoshida, T. Mizoguchi, and Y. Hatsugai, arXiv:1912.12022.

[26] M. C. Rechtsman, J. M. Zeuner, Y. Plotnik, Y. Lumer, D. Podolsky, F. Dreisow, S. Nolte, M. Segev, and A. Szameit, Nature (London) 496, 196 (2013). 
[27] M. Hafezi, S. Mittal, J. Fan, A. Migdall, and J. M. Taylor, Nat. Photon. 7, 1001 (2013).

[28] L. J. Maczewsky, J. M. Zeuner, S. Nolte, and A. Szameit, Nat. Commun. 8, 13756 (2017).

[29] A. E. Hassan, F. K. Kunst, A. Moritz, G. Andler, E. J. Bergholtz, and M. Bourennane, Nat. Photon. 13, 697 (2019).

[30] E. J. Bergholtz, J. C. Budich, and F. K. Kunst, arXiv:1912.10048.

[31] T. Schwartz, G. Bartal, S. Fishman, and M. Segev, Nature (London) 446, 52 (2007).

[32] O. Peleg, G. Bartal, B. Freedman, O. Manela, M. Segev, and D. N. Christodoulides, Phys. Rev. Lett. 98, 103901 (2007).

[33] A. Szameit, M. C. Rechtsman, O. Bahat-Treidel, and M. Segev, Phys. Rev. A 84, 021806(R) (2011).

[34] O. Bahat-Treidel, O. Peleg, and M. Segev, Opt. Lett. 33, 2251 (2008).

[35] Q. Liang, Y. Yan, and J. Dong, Opt. Lett. 36, 2513 (2011).

[36] Y. V. Kartashov, J. M. Zeuner, A. Szameit, V. A. Vysloukh, and L. Torner, Opt. Lett. 38, 3727 (2013).

[37] Y. Plotnik, M. C. Rechtsman, D. Song, M. Heinrich, J. M. Zeuner, S. Nolte, Y. Lumer, N. Malkova, J. Xu, A. Szameit, Z. Chen, and M. Segev, Nat. Mater. 13, 57 (2014).

[38] Y.-J. Wu and J. Hou, Phys. Rev. A 99, 062107 (2019).

[39] M. I. Katsnelson, Mater. Today 10, 20 (2007).

[40] A. K. Geim and K. S. Novoselov, Nat. Mater. 6, 183 (2007).

[41] A. H. Castro Neto, F. Guinea, N. M. R. Peres, K. S. Novoselov, and A. K. Geim, Rev. Mod. Phys. 81, 109 (2009).

[42] A. K. Geim, Science 324, 1530 (2009).

[43] M. I. Katsnelson, Graphene: Carbon in Two Dimensions (Cambridge University Press, Cambridge, 2012).

[44] T. O. Wehling, A. M. Black-Schaffer, and A. V. Balatsky, Adv. Phys. 63, 1 (2014).

[45] M. Z. Hasan and C. L. Kane, Rev. Mod. Phys. 82, 3045 (2010).

[46] X.-L. Qi and S.-C. Zhang, Rev. Mod. Phys. 83, 1057 (2011).

[47] M. Z. Hasan and J. E. Moore, Annu. Rev. Condens. Matter Phys. 2, 55 (2011).

[48] B. A. Bernevig and T. L. Hughes, Topological Insulators and Topological Superconductors (Princeton University Press, Princeton, 2013).

[49] B. Yan and C. Felser, Annu. Rev. Condens. Matter Phys. 8, 337 (2017).

[50] M. Z. Hasan, S.-Y. Xu, I. Belopolski, and C.-M. Huang, Annu. Rev. Condens. Mattter Phys. 8, 289 (2017).

[51] N. P. Armitage, E. J. Mele, and A. Vishwanath, Rev. Mod. Phys. 90, 015001 (2018).
[52] A. V. Balatsky, I. Vekhter, and J.-X. Zhu, Rev. Mod. Phys. 78, 373 (2006).

[53] T. O. Wehling, M. I. Katsnelson, and A. I. Lichtenstein, Chem. Phys. Lett. 476, 125 (2009).

[54] Y. V. Skrypnyk and V. M. Loktev, Low Temp. Phys. 44, 1112 (2018).

[55] I. M. Lifshitz, Adv. Phys. 13, 483 (1964).

[56] I. M. Lifshitz, Phys. Usp. 7, 549 (1965).

[57] V. M. Pereira, F. Guinea, J. M. B. Lopes dos Santos, N. M. R. Peres, and A. H. Castro Neto, Phys. Rev. Lett. 96, 036801 (2006).

[58] N. M. R. Peres, F. Guinea, and A. H. Castro Neto, Phys. Rev. B 73, 125411 (2006).

[59] Yu. V. Skrypnyk and V. M. Loktev, Phys. Rev. B 73, 241402(R) (2006).

[60] T. O. Wehling, A. V. Balatsky, M. I. Katsnelson, A. I. Lichtenstein, K. Scharnberg, and R. Wiesendanger, Phys. Rev. B 75, 125425 (2007).

[61] T. O. Wehling, S. Yuan, A. I. Lichtenstein, A. K. Geim, and M. I. Katsnelson, Phys. Rev. Lett. 105, 056802 (2010).

[62] Y. C. Chang and S. Haas, Phys. Rev. B 83, 085406 (2011).

[63] M. M. Ugeda, I. Brihuega, F. Guinea, and J. M. GómezRodríguez, Phys. Rev. Lett. 104, 096804 (2010).

[64] K. K. Gomes, W. Mar, W. Ko, F. Guinea, and H. C. Manoharan, Nature (London) 483, 306 (2012).

[65] Y. Jiang, P.-W. Lo, D. May, G. Li, G.-Y. Guo, F. B. Anders, T. Taniguchi, K. Watanabe, J. Mao, and E. Y. Andrei, Nat. Commun. 9, 2349 (2018).

[66] X. Wen-Yan, Z. Li-Zhi, H. Li, Q. Yan-De, W. Ye-Liang, L. Xiao, and D. Shi-Xuan, Chin. Phys. B 28, 046801 (2019).

[67] O. V. Yazyev and L. Helm, Phys. Rev. B 75, 125408 (2007).

[68] O. V. Yazyev, Rep. Prog. Phys. 73, 056501 (2010).

[69] A. F. Tzortzakakis, K. G. Makris, and E. N. Economou, Phys. Rev. B 101, 014202 (2020).

[70] Y. Huang and B. I. Shklovskii, Phys. Rev. B 101, 014204 (2020).

[71] G. Bartal, O. Cohen, H. Buljan, J. W. Fleischer, O. Manela, and M. Segev, Phys. Rev. Lett. 94, 163902 (2005).

[72] K. L. Zhang, X. M. Yang, and Z. Song, Phys. Rev. B 100, 024305 (2019).

[73] H. Xue, Q. Wang, B. Zhang, and Y. D. Chong, arXiv:1912.10937.

[74] S. Doniach and E. H. Sondheimer, Green's Functions for Solid State Physicists (World Scientific, Singapore, 1988). 